\title{
Funcionalidad familiar en la depresión de adolescentes de la Institución Educativa Particular "Gran Amauta de Motupe" Lima, 2018
}

Family functionality in the depression of adolescents in the private educational institution "Gran Amauta de Motupe” Lima, 2018

Andy Nataly Olivera ${ }^{1}$, Edith Gissela Rivera², Milagros Gutiérrez Trevejo ${ }^{3}$, Juan Méndez

\section{RESUMEN}

Objetivo: Determinar la influencia de la funcionalidad familiar en la depresión de adolescentes de la institución educativa particular "Gran Amauta de Motupe” Lima, 2018. Material y método: El diseño utilizado fue no experimental, de corte transversal, correlacional causal, los instrumentos utilizados fueron: la Escala de Evaluación de Cohesión y Adaptabilidad familiar, FACES IV, de Olson y el Inventario de Depresión de Beck (IDB-II). Resultados: La muestra estuvo compuesta por 91 adolescentes de primero a quinto de secundaria entre 12 y 17 años de edad de nivel secundario. El análisis general mostró un 51.6\% de depresión mínima, 28.5\% leve, $10.9 \%$ moderada y $8.7 \%$ severa. Mientras que la funcionalidad familiar mostró $65.9 \%$ de funcionalidad intermedia y 34\% baja. Conclusión: Los adolescentes que presentan menor funcionalidad familiar tienden a presentar mayor sintomatología depresiva.

PALABRAS CLAVES: Adolescente, depresión, funcionalidad familiar (DeCS, BIREME).

Psicóloga. Maestra en Gestión de los servicios de la salud. Psicóloga asistente, penal San Juan de Lurigancho. Lima, Perú.

Profesora de educación primaria y problemas de aprendizaje. Magister en administración de la educación. Docente en investigación, Universidad César vallejo, Universidad Privada Norbert Wiener. Lima, Perú.

Estudiante del Internado Facultad de Psicología, Universidad Peruana Cayetano Heredia. Lima, Perú.

Psicólogo. Profesor de psicología. Especialista en Audición, Lenguaje y Aprendizaje. Doctor en psicología. Docente de investigación, Universidad, César Vallejo. Lima, Perú. 


\section{SUMMARY}

Objective: Determine the influence of family functionality on adolescent depression at the private educational institution “Gran Amauta de Motupe” Lima, 2018. Material and method: The design used was non-experimental, cross-sectional, causal correlational, the instruments used were : the Family Cohesion and Adaptability Assessment Scale, FACES IV, by Olson and the Beck Depression Inventory (IDB-II). Results: The sample consisted of 91 adolescents from first to fifth grade of secondary school between 12 and 17 years of age of secondary level. The general analysis showed a 51.6\% minimum depression, $28.5 \%$ mild, $10.9 \%$ moderate and $8.7 \%$ severe. While the family functionality showed $65.9 \%$ intermediate functionality and $34 \%$ low. Conclusion: Adolescents with lower family functionality tend to have greater depressive symptomatology.

\section{KEYWORDS: Adolescent, depression, family functionality (DeCS, BIREME).}

\section{INTRODUCCIÓN}

La depresión es un trastorno mental frecuente que afecta a más de 322 millones de personas a nivel mundial, correspondiendo 48.16 millones a las Américas. Este trastorno provoca alrededor de 800 mil suicidios anuales, constituyéndose en la segunda causa de muerte en el grupo etario de 15 a 29 años. Se estima y predice, que esta cifra aumentará que para el año 2020 será la segunda causa de pérdida de más años de vida saludable (1). Su presencia suele estar asociada a una mayor comorbilidad, existiendo el riesgo de presentar algún tipo de trastorno o dependencia hacia las drogas (2). Los problemas de salud mental en general y la depresión en particular tienden a hacer su aparición a finales de la infancia e inicios de la adolescencia, constituyéndose en una de las más importantes causas de morbilidad en los jóvenes en el mundo (1).

Los factores que pueden causar depresión en la familia, son las relaciones inadecuadas entre sus integrantes, que puede llevar al adolescente a perder el sentido de la vida y comenzar a desarrollar ideas suicidas para suprimir el dolor emocional por el que atraviesa. Por este motivo, los desajustes emocionales que ellos presentan están estrechamente relacionados a las contradicciones familiares(4).

En el Perú, la población adolescente comprendida entre 10 y 19 años, representando el 20\% del total de la población, de los cuales, el Ministerio de Salud Minsa en el 2016 reportó aproximadamente 2 millones de casos de depresión y ansiedad vinculados con conflictos familiares, de las cuales el $70 \%$ de pacientes fueron niños y jóvenes menores de 18 años (5). Diversos reportes de investigación de la realidad peruana demuestra la prevalencia de trastornos depresivos asociados a ideas suicidas y conflictos familiares. Desde 2012 se reportaron que el 20\% a $25 \%$ de adolescentes tenían deseos de morir y un $10 \%$ a $15 \%$ intentó llevar a cabo su intensión. Estas conductas se explicarían por la creciente violencia familiar, puesto que en la mayoría de casos (entre 60\% a 70\%) detectados con depresión e ideas suicidas estarían ligados a problemas con los padres (5). Además, a menor funcionamiento familiar mayor ideación suicida y viceversa (4).

La depresión de los adolescentes se explicaría en parte por la exposición de los adolescentes a conflictos familiares hostiles, que no son congruentes y que muestran patrones de educación autoritarios, los relaciona con diversos desajustes emocionales y cognitivos, en la cual aparece la depresión, debido a que la construcción de la personalidad de los jóvenes está relacionada con los modelos parentales de crianza y educación y mostrando una correlación negativa moderada entre la depresión y la cohesión familiar en los evaluados. $(6,8)$, que se corrobora con un estudio entre la cohesión familiar y nivel de sintomatología depresiva en adolescentes que mostró una relación negativa entre la presencia de sintomatología depresiva y el nivel de cohesión familiar existiendo una correlación significativa entre las dos variables que evidenciaban altos índices de sintomatología depresiva (6). Además, la dinámica del grupo familiar influye de manera directa o indirecta sobre las conductas suicidas de un miembro del grupo familiar (9). Sin embargo, un estudio demostró que no existían diferencias estadísticamente significativas al relacionar los niveles de depresión con funcionalidad familiar (9). 
El objetivo fue determinar la influencia de la funcionalidad familiar en la depresión de adolescentes de la Institución Educativa Particular "Gran Amauta de Motupe” de Lima.

El estudio se justifica en la medida que permita asumir acciones futuras de prevención e intervención en el problema de la depresión en adolescentes y la acción en las familias tanto desde el campo de la salud como de la educación.

\section{MATERIAL Y MÉTODOS}

Se realizó un estudio cuantitativo(11), no experimental (11), de corte transversal, correlacional causal (11). La muestra intencional estuvo conformada por 91 adolescentes entre los 12 a 17 años de edad pertenecientes a la Institución Educativa Particular "Gran Amauta de Motupe”.

Para la evaluación de las variables de estudio se utilizaron dos pruebas: (a) la Escala de Evaluación de Cohesión y Adaptabilidad familiar (FACES IV) de Olson para hallar la funcionalidad familiar y sus dimensiones: cohesión, adaptabilidad, comunicación y satisfacción, (b) el Inventario de Depresión de Beck (IDB-II) para medir el nivel de depresión general, somática y cognitivo-afectiva. Antes aplicar los instrumentos, se constató la confiabilidad de ambos instrumentos en una muestra piloto. Los resultados mostraron una confiabilidad Alfa de Cronbach de 0.81 para el FACES IV y de 0.89 para el Inventario de depresión de Beck II. Se determinó que los instrumentos tenían un alto nivel de confiabilidad que permitirían la medición objetiva de las variables.
Para el análisis estadístico descriptivo se utilizaron las tablas de contingencia y para la prueba de las hipótesis se utilizó la prueba de regresión logística multinomial, teniendo en consideración que la variable en estudio era cualitativa de escala ordinal.

El protocolo de investigación fue revisado y aprobado por Dirección del Centro Educativo y por el comisión de investigación de la Universidad César Vallejo. Los participantes fueron informados que los cuestionarios cumplimentados tenían carácter anónimo que protege el derecho a la identidad y dignidad.

\section{RESULTADOS}

Se evaluaron 91 adolescentes, de primero a quinto de secundaria entre 12 y 17 años de edad de nivel secundario: 46 (51\%) del sexo femenino y 45 (49\%) del sexo masculino. La distribución de la muestra por grados fue: primer grado 28 (31\%), segundo 18 (20\%), tercero 12 (13\%), cuarto $16(17 \%)$ y quinto 17 (19\%).

Al comparar los niveles de depresión con los niveles de la funcionalidad familiar, se obtuvieron los siguientes resultados:

De los 91 sujetos evaluados, el 51,6\% tuvo depresión mínima, 28,6\% depresión leve, 11\% depresión moderada y $8.8 \%$ depresión severa. Además, de los 31 sujetos que mostraron funcionalidad baja, el 35,5\% tuvo depresión mínima, 32,3\% depresión leve, 16,1\% depresión moderada y 16,1\% depresión severa. Por otra parte, de los 60 sujetos que mostraron funcionalidad intermedia, el 60\% tuvo depresión mínima, 26,7\% depresión leve, 8,3\% depresión

Tabla 1. Niveles de la Funcionalidad Familiar y de la Depresión de adolescentes de la Institución Educativa Particular "Gran Amauta de Motupe"

\begin{tabular}{|c|c|c|c|c|c|}
\hline \multicolumn{2}{|c|}{ Nivel de Funcionalidad Familiar } & \multicolumn{4}{|c|}{ Nivel de Depresión } \\
\hline \multicolumn{2}{|c|}{ Depresión Mínima } & $\begin{array}{l}\text { Depresión } \\
\text { Leve }\end{array}$ & $\begin{array}{l}\text { Depresión } \\
\text { Moderada }\end{array}$ & $\begin{array}{c}\text { Depresión } \\
\text { Severa }\end{array}$ & \\
\hline \multirow{2}{*}{ Funcionalidad Baja } & Recuento & 11 & 10 & 5 & 5 \\
\hline & $\%$ & $35.5 \%$ & $32.3 \%$ & $16.1 \%$ & $16.1 \%$ \\
\hline \multirow{2}{*}{ Funcionalidad Intermedia } & Recuento & 36 & 16 & 5 & 3 \\
\hline & $\%$ & $60.0 \%$ & $26.7 \%$ & $8,3 \%$ & $5.0 \%$ \\
\hline \multirow{2}{*}{ Total } & Recuento & 47 & 26 & 10 & 8 \\
\hline & $\%$ & $51.6 \%$ & $28.6 \%$ & $11.0 \%$ & $8.8 \%$ \\
\hline
\end{tabular}


Tabla 2. Cohesión Familiar y de los niveles de la Depresión de adolescentes de la Institución Educativa Particular "Gran Amauta de Motupe"

\begin{tabular}{|c|c|c|c|c|c|}
\hline \multirow{2}{*}{\multicolumn{2}{|c|}{ Nivel de Cohesión Familiar }} & \multicolumn{4}{|c|}{ Nivel de Depresión } \\
\hline & & $\begin{array}{l}\text { Depresión } \\
\text { Mínima }\end{array}$ & $\begin{array}{l}\text { Depresión } \\
\text { Leve }\end{array}$ & $\begin{array}{l}\text { Depresión } \\
\text { Moderada }\end{array}$ & $\begin{array}{c}\text { Depresión } \\
\text { Severa }\end{array}$ \\
\hline \multirow{2}{*}{ Baja } & Recuento & 18 & 9 & 6 & 5 \\
\hline & $\%$ & $47.4 \%$ & $23.7 \%$ & $15.8 \%$ & $13.2 \%$ \\
\hline \multirow{2}{*}{ Intermedia } & Recuento & 15 & 5 & 2 & 2 \\
\hline & $\%$ & $62.5 \%$ & $20.8 \%$ & $8.3 \%$ & $8.3 \%$ \\
\hline \multirow{2}{*}{ Alta } & Recuento & 14 & 12 & 2 & 1 \\
\hline & $\%$ & $48.3 \%$ & $41.4 \%$ & $6.9 \%$ & $3.4 \%$ \\
\hline \multirow{2}{*}{ Total } & Recuento & 47 & 26 & 10 & 8 \\
\hline & $\%$ & $51.6 \%$ & $28.6 \%$ & $11.0 \%$ & $8.8 \%$ \\
\hline
\end{tabular}

Tabla 3. Adaptabilidad Familiar y de los niveles de la Depresión de adolescentes de la Institución Educativa Particular "Gran Amauta de Motupe"

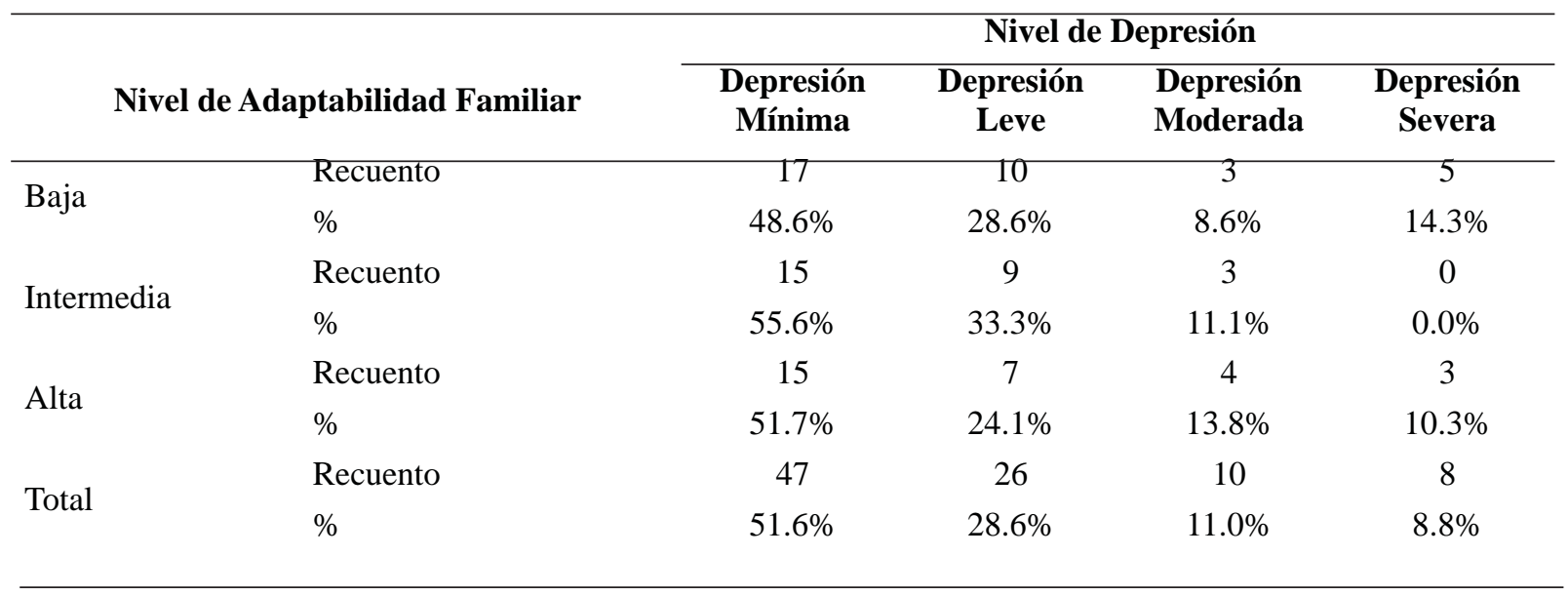

moderada y 5\% depresión severa. Se concluyó que, a menor nivel en la funcionalidad familiar habría mayor nivel de depresión en los adolescentes evaluados (tabla 1).

Al comparar la asociación entre la cohesión familiar y los niveles de depresión se encontró que de los 38 sujetos que tenían cohesión familiar baja, 47,4\% mostraron depresión mínima, 23,7\% depresión leve, 15,8\% depresión moderada y 13,2\% depresión severa. Por otra parte, de los 22 sujetos investigados que indicaron cohesión familiar intermedia, 62,5\% mostraron depresión mínima, 20,8\% depresión leve, 8,3\% depresión moderada y $8,3 \%$ depresión severa. Además, de los 29 investigados que indicaron cohesión familiar alta, 48,3\% mostraron depresión mínima, 41,4\% depresión leve, 6,9\% depresión moderada y 3,4\% depresión severa.
En conclusión, a menor cohesión familiar mayor depresión y viceversa (tabla 2).

Al comparar la asociación entre la adaptabilidad Familiar y de los niveles de la Depresión de adolescentes de la Institución Educativa Particular “Gran Amauta de Motupe” se encontró que a más baja adaptabilidad mayor depresión de los investigados (tabla 3). Sin embargo, esta asociación inversa entre la comunicación familiar, la satisfacción familiar y la depresión es más evidente que con la cohesión y la adaptabilidad familiar (tablas 4, 5).

La contrastación de las dimensiones comunicación familiar y la satisfacción familiar son dos variables que mostraron una mayor incidencia en los niveles de depresión del grupo investigado (tablas 4,5). 
Tabla 4: Comunicación Familiar y de los niveles de la Depresión de adolescentes de la Institución Educativa Particular "Gran Amauta de Motupe"

\begin{tabular}{llcccc}
\hline & & \multicolumn{3}{c}{ Nivel de Depresión } \\
\cline { 3 - 6 } Nivel de Comunicación Familiar & $\begin{array}{c}\text { Depresión } \\
\text { Mínima }\end{array}$ & $\begin{array}{c}\text { Depresión } \\
\text { Leve }\end{array}$ & $\begin{array}{c}\text { Depresión } \\
\text { Moderada }\end{array}$ & $\begin{array}{c}\text { Depresión } \\
\text { Severa }\end{array}$ \\
\hline \multirow{2}{*}{ Baja } & Recuento & 11 & 9 & 6 & 6 \\
& $\%$ & $34.4 \%$ & $28.1 \%$ & $18.8 \%$ & $18.8 \%$ \\
Intermedia & Recuento & 20 & 10 & 3 & 1 \\
& $\%$ & $58.8 \%$ & $29.4 \%$ & $8.8 \%$ & $2.9 \%$ \\
Alta & Recuento & 16 & 7 & 1 & 1 \\
& $\%$ & $64.0 \%$ & $28.0 \%$ & $4,0 \% 0^{\mathrm{a}}$ & $4.0 \%$ \\
Total & Recuento & 47 & 26 & 10 & 8 \\
& $\%$ & $51.6 \%$ & $28.6 \%$ & $11.0 \%$ & $8.8 \%$ \\
\hline
\end{tabular}

Tabla 5: Satisfacción Familiar y de los niveles de la Depresión de adolescentes de la Institución Educativa Particular "Gran Amauta de Motupe"

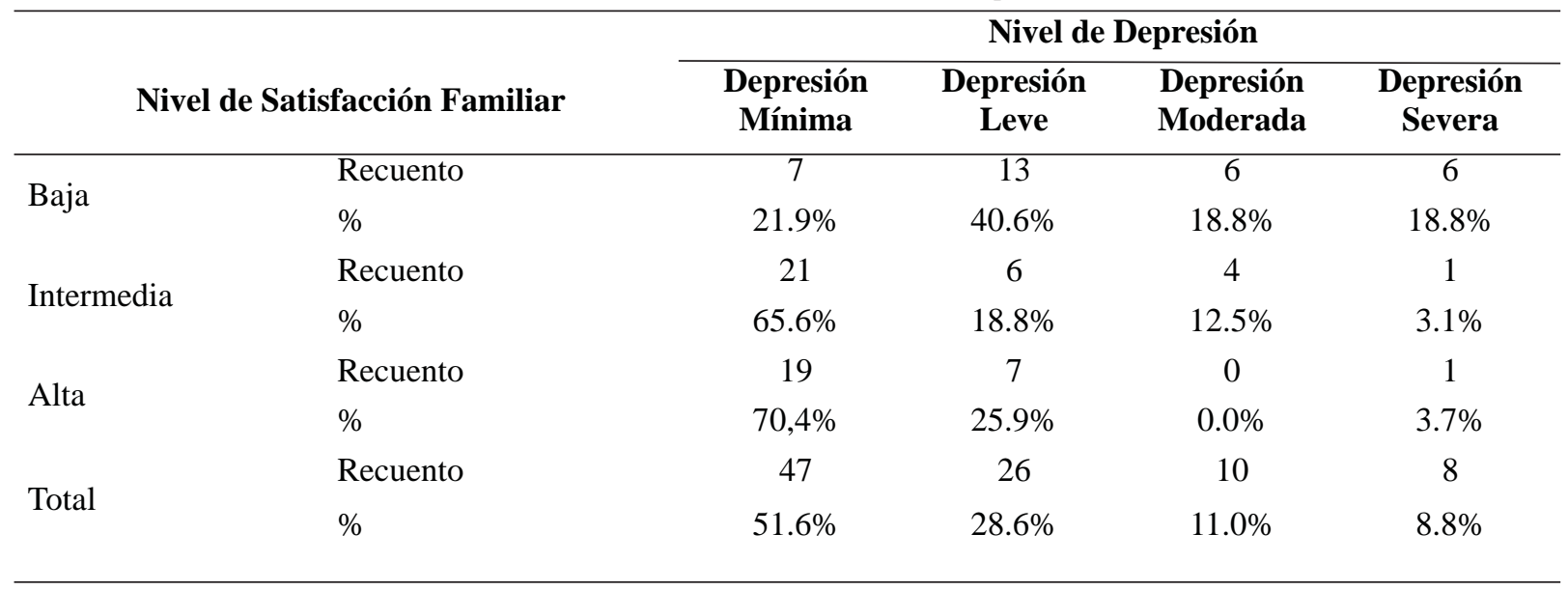

Tabla 6: Determinación del ajuste de los datos de la funcionalidad familiar en la depresión de adolescentes de la Institución Educativa Particular "Gran Amauta de Motupe”

\begin{tabular}{|c|c|c|c|c|}
\hline \multirow{2}{*}{ Modelo } & \multirow{2}{*}{$\begin{array}{c}\text { Criterios de ajuste de } \\
\text { modelo } \\
\text { Logaritmo de la } \\
\text { verosimilitud -2 }\end{array}$} & \multicolumn{3}{|c|}{$\begin{array}{c}\text { Pruebas de la razón de } \\
\text { verosimilitud }\end{array}$} \\
\hline & & Chi-cuadrado & gl & Sig. \\
\hline Sólo intersección & 174,379 & & & \\
\hline Final & 164,687 & 9,692 & 3 & 021 \\
\hline
\end{tabular}

La contrastación de las hipótesis estadísticas demostró que el valor de Chi cuadrado 9,692 mayor a 0 y el p valor (sig.) es igual a 0.021 siendo esta menor a 0,05 lo que indicaría el rechazo de la hipótesis nula, las variables de estudio no son independientes la una de la otra, por la cual se acepta la hipótesis de alterna, que indica que si existe influencia significativa de la funcionalidad familiar en la depresión en los adolescentes de la Institución Educativa Particular "Gran Amauta de Motupe". Por lo que se infiere 
que la funcionalidad familiar influye en la presencia depresión en los adolescentes (tabla 6).

\section{DISCUSIÓN}

En presente estudio, pretendió medir la influencia de las variables funcionalidad familiar en la depresión de los adolescentes de una Institución Educativa particular. Al investigar sobre el funcionamiento familiar en la depresión de adolescentes, se pudo tomar en cuenta que la funcionabilidad familiar no solo depende de cómo reaccionan los hijos sino también de la forma en cómo los padres o apoderados del menor lo perciben, comprenden y asumen esta etapa de la vida. En esta investigación el resultado indica que si existe influencia significativa de la funcionalidad familiar en la depresión de adolescentes. Asimismo, respecto a las dimensiones de la funcionalidad familiar en la depresión, se encontró que no existe influencia significativa de las dimensiones cohesión y adaptabilidad familiar en la depresión de adolescentes, sin embargo, si se encontró influencia significativa en las dimensiones comunicación y satisfacción familiar en la depresión de adolescentes.

En relación a los resultados de esta investigación, estos coinciden con los obtenidos por Torrel y Delgado, quienes encontraron una asociación significativa entre las variables funcionalidad familiar y la depresión. Por la cual se comprueba la hipótesis de trabajo, puesto que los adolescentes que presentan mayores niveles de depresión, perciben mayores conflictos familiares (8). Asimismo, coincide con lo hallado por Álvarez, Ramírez, Silva, Coffin, \& Jiménez, que demostraron que existe una correlación negativa moderada entre la depresión y la cohesión familiar en los evaluados (6).

Sin embargo, en el estudio llevado a cabo por Salinas, Alonso, Quintero, Hernández, Molina, Lozada, et al. mostraron que no existían diferencias estadísticamente significativas al relacionar los niveles de depresión con funcionalidad familiar (10). Por lo que se puede inferir en torno a las variables de estudio, la funcionalidad familiar y depresión en adolescentes tienden a tener una reacción diferente dependiendo a cada realidad o cultura al cual pertenezca el individuo. En referente a la familia Vargas se consideró que ésta juega un papel fundamental, siendo un factor protector y de apoyo indispensable para el adolescente, una buena funcionalidad familiar permitirá construir estrategia de afrontamiento hacia diversos factores de riesgo que pueden generar vulnerabilidad con el tiempo, pudiendo repercutir en su calidad de vida, presentando alguna serie de alteraciones o trastornos de estado de ánimo (12).

De acuerdo a lo anterior, se puede inferir que el contexto familiar influye de manera significativa en las personas durante su adolescencia, debido a que interviene en la forma en que se enfrentan las situaciones cotidianas (6). La familia como grupo dinámico y flexible, está en constante trasformación, que implica el desarrollo de las capacidades de adaptación a diversas situaciones, que tienen como influencia al contexto externo y a los cambios propios de la familia (6). Por ello se infiriere que los resultados, dan a conocer que las carencias de redes de apoyo en la dinámica familiar de los adolescentes, afectan en su desarrollo en torno a los procesos psicosociales, siendo esto peligroso, debido a que es un factor de riesgo para la depresión u algún otro tipo de conductas autodestructivas, el modo como el adolescente exprese y reciba afecto tiene influencia para la aparición de depresión en nuestro contexto. La familia es el factor protector que tiene el ser humano y esta puede convertirse en factor de riesgo cuando surgen problemas dentro de ella. Actualmente existen muchas familias que ponen en peligro su capacidad de funcionamiento y de supervivencia, lo cual se torna un factor de riesgo para el desarrollo normal del adolescente. Lo presentado en este estudio, al igual que lo encontrado en la literatura, nos indica que la funcionalidad familiar, la relación entre padres e hijos y los recursos afectivos dentro del sistema familiar, están estrechamente relacionados con la sintomatología depresiva en los adolescentes.

En conclusión, el estudio realizado con los adolescentes de la institución educativa "Gran Amauta de Motupe” en el 2018, demostró que existía influencia significativa de la funcionalidad familiar en la depresión de los adolescentes, por lo que se infiere que la funcionalidad familiar influye en la aparición de depresión en los adolescentes. Asimismo, se evidenció que existía influencia significativa en las dimensiones comunicación y satisfacción familiar en la depresión de adolescentes. Sin embargo, cabe señalar que se no evidenció influencia significativa de las dimensiones cohesión y adaptabilidad familiar en la depresión. Sin embargo, los resultados de esta 
investigación solo son válidos para el ámbito en donde se desarrolló, debido al tamaño y al tipo de muestra con la que se trabajó.

\section{Correspondencia:}

Andy Nataly Olivera

Correo electrónico: andyolivera12@gmail.com

\section{REFERENCIAS BIBLIOGRÁFICAS}

1. Organización Mundial de la Salud. (2017) Depresión y otros trastornos mentales comunes. Estimaciones sanitarias mundiales. URL disponible en: iris. paho.org/xmlui/bitstream/handle/ 123456789/34006/ PAHONMH17005-spa.pdf (Fecha de acceso: 22 de enero del 2018)

2. Hernández, A. Leyva, R. López, V. y Nava, G. Depresión en adolescentes y funcionamiento familiar. Revista Médica del Instituto Mexicano del Seguro Social. 2006; 45(3): 225-232. URL disponible en: http://www.medigraphic.com/pdfs/imss/im-2007/ im073d.pdf (Fecha de acceso: 7 de diciembre del 2017)

3. Organización Mundial de la Salud. Depresión. 2018. URL disponible en: http://www.who.int/es/newsroom/fact-sheets/detail/depression (Fecha de acceso: 9 de marzo del 2018)

4. Pérez, P. Funcionamiento familiar e ideación suicida en alumnos de 5to año de educación Secundaria del distrito de San Juan de Miraflores. Psique Mag. 2016; 4(1): 81-93. URL disponible en: http://ojs.ucvlima. edu.pe/index.php/psiquemag/article/view/136(Fechade acceso: 2 de marzo del 2018)

5. Sausa, M. (03/02/2018) Menores ocupan el 70\% de atenciones en salud mental [INFOGRAFÍA]. Perú 21. URL disponible en: https://peru21.pe/peru/ cifras-salud-mental-peru-menores-ocupan-70atenciones-infografia-394376 (Fecha de acceso: 23 de julio del 2018)
6. Álvarez, M., Ramírez, B., Silva, A., Coffin, N., y Jiménez, M. La relación entre depresión y conflictos familiares en adolescentes. International Journal of Psychology and Psychological Therapy. 2009; 9(2): 205-210. URL disponible en: http://www.redalyc.org/ html/ 560/560128 78005/ (Fecha de acceso: 25 de enero del 2018)

7. Buitrago, J., Archila, P., Yasmith, L., y GüicháDuitama, Á. Relación entre sintomatología depresiva y cohesión familiar en adolescentes de una institución educativa de Boyacá. Psicogente. (2017); 20(38): 296307. URL disponible en: http://dx.doi.org/10.17081/ psico. (Fecha de acceso: 27 de enero del 2018)

8. Torrel, M., y Delgado, M. Funcionamiento familiar y depresión en adolescentes de la IE ZarumillaTumbes, 2013. Ciencia y desarrollo. 2016; 17(1): 47-53. DOI: http://dx.doi.org/10.21503/CienciayDesarrollo. 2014.v17i1.06 (Fecha de acceso: 25 de enero del 2018)

9. Burgos, G. V., Narváez, N. N., de las Mercedes Bustamante, P. S., Burrone, M. S., Fernández, R., y Abeldano, R. A. Funcionamiento familiar e intentos de suicidio en un hospital público de Argentina. Acta de Investigación Psicológica. 2017; 7(3): 2802-2810. DOI: $\quad$ https://doi.org/10.1016/j.aipprr.2017.11.009 (Fecha de acceso: 16 de enero del 2018)

10. Salinas, A., Alonso, S., Quintero, et al. Relación entre depresión y funcionalidad familiar en adolescentes de instituciones educativas públicas y privadas Municipio Libertador. Merida-Venezuela. Revista Academia. 2013; 12(28): 317-324. URL disponible en: erevistas. saber.ula. ve/index.php/academia/ rticle/view/6168 (Fecha de acceso: 6 de febrero del 2018)

11. Hernández, R., Fernández, C. y Baptista, P. Metodología de la Investigación. 6ta. ed. México DF: Mc Graw-Hill; 2014. pp. 4; 93; 152.

12. Vargas, H. Tipo de familia y ansiedad y depresión. Rev Med Hered. 2014; 25:57-59. URL disponible en: http:// www.scielo.org.pe/pdf/rmh/v25n2/v25n2e1.pdf (Fecha de acceso: 6 de febrero del 2018).
Recibido: 16-01-2019

Aceptado: 29-04-2019 